\title{
REPRESENTATION OF THE SHORT-RANGE INTERACTIONS IN LIQUID HELIUM VIA MODIFIED HARD SPHERE POTENTIALS
}

\author{
I. O. Vakarchuk, A. A. Rovenchak \\ Ivan Franko National University of Lviv, Department for Theoretical Physics \\ 12 Drahomanov Str., Lviv, UA-79005, Ukraine
}

(Received March 31, 2001; received in final form July 9, 2001)

\begin{abstract}
In this paper we propose five different modifications of the hard sphere potential for the modeling of a short-range repulsion and the calculation of thermodynamic and transport properties of liquid ${ }^{4} \mathrm{He}$. We calculate the potential energy, the total energy, and the sound velocity at $T=0 \mathrm{~K}$. It is shown that three of the proposed potentials give a satisfactory description of these properties.

Keywords: liquid helium, interatomic potential, short-range interaction, thermodynamic properties, sound velocity.
\end{abstract}

PACS numbers: 34.20.Cf, 67.40.Kh, 67.40.Mj

\section{INTRODUCTION}

In this paper we calculate some properties of the helium system using the modifications of helium-helium interatomic potential obtained in [1].

The method of receiving the potential is based on the collective variables formalism as described in our previous work [1].

Numerical methods could be grouped in two large parts: 1) calculations with adjustable parameters using experimental data on thermodynamic and transport properties [2-5], 2) ab initio quantum-mechanical computations (mainly Monte Carlo) [6,7]. A good comparison between various potentials, calculated properties of helium, and the experimental data might be found in [8].

We analyze our potentials due to the form of the potential curve and such properties as the potential energy, the total energy, and the sound velocity, all calculated at $T=0 \mathrm{~K}$, i. e., for the ground state.

Concerning the form of the potential curve, one can notice that the short-range part of the potential is not very significant starting from distances about $2 \AA$ and less. This statement becomes clear if one looks at the pair distribution function $F_{2}(R)$ of helium [9]: $F_{2}(R<$ $2.212 \AA)=0$.

On the other hand, the results for the potential from [1] lead to the value $-7.8 \mathrm{~K}$ for the total energy of the system of helium atoms at $0 \mathrm{~K}$, while the experiment gives $-7.17 \mathrm{~K}$ [10], we consider it as a good agreement. But we obtained the value of the potential energy $-15.5 \mathrm{~K}$, and computer simulations [8] predict it in the range from about -20 to $-22 \mathrm{~K}$ (there is no experimental measurements available for this quantity). This difference may not seem to be quite satisfactory if one calculates quantities connected with the potential energy only.

Thus, we tried to correct our potential for it could reflect the short-range interactions more precisely unlike that obtained in [1]. For this purpose we used some modification of the hard sphere potential.

The paper is organized as follows. The calculating procedure is described in Section 2. Different forms for the short-range repulsion are presented in Section 3 , the respective potential curves are also given there. In Section 4 we adduce the computation of the energy and the sound velocity of ${ }^{4} \mathrm{He}$ in the ground state.

\section{CALCULATING PROCEDURE}

The following relation was used for the calculation of the potential Fourier image $\nu_{q}$, see $[1,11]$ :

$$
\begin{aligned}
\frac{N}{V} \frac{m \nu_{q}}{\hbar^{2}} & +\mathbf{q}^{2} a_{2}(\mathbf{q})-\mathbf{q}^{2} a_{2}^{2}(\mathbf{q}) \\
& -\frac{1}{2 N} \sum_{\mathbf{k} \neq \mathbf{0}} \mathbf{k}^{2} a_{4}(\mathbf{k},-\mathbf{k}, \mathbf{q},-\mathbf{q}) \\
& +\frac{1}{N} \sum_{\mathbf{k} \neq \mathbf{0}}(-\mathbf{k}-\mathbf{q}) \mathbf{k} a_{3}(\mathbf{q}, \mathbf{k},-\mathbf{k}-\mathbf{q})=0
\end{aligned}
$$

$N$ is the total number of particles, $V$ is the volume of the system, in the thermodynamic limit we have $N / V=$ const $=\rho=0.02185 \tilde{\mathrm{A}}^{-3} ; m$ is the mass of ${ }^{4} \mathrm{He}$ atom; the quantities $a_{3}, a_{4}$ are expressed through $a_{2}$, see $[1,11]$.

We are going to solve this problem in the approximation of "two sums over the wave vector". Such calculations were made in [1] with the expression for $a_{2}$ in the form of

$$
a_{2}(\mathbf{q})=\frac{1}{2}\left(1-\frac{1}{S_{q}}\right)-\frac{1}{2} \Sigma(\mathbf{q})
$$

$\Sigma(\mathbf{q})$ being complex functional of $a_{2}$ containing summation (integration) over the wave vector $[1,11]$. 
The equation (1) for $\nu_{q}$ is solved using iterative procedure. We have used the corrected expression for the zeroth approximation of the quantity $a_{2}[12]$ :

$$
a_{2}^{0}(\mathbf{q})=\frac{1}{2}\left(\frac{1}{S_{q}^{0}}-\frac{1}{S_{q}}\right),
$$

while in [1] we used

$$
a_{2}^{0}(\mathbf{q})=\frac{1}{2}\left(1-\frac{1}{S_{q}}\right)
$$

$S_{q}^{0}$ stands for the short-range structure factor, i. e. it corresponds to the short-range repulsive interactions. The question obtaining this structure factor $S_{q}^{0}$ is elucidated in the next section.

\section{SHORT-RANGE POTENTIAL}

\section{A. Hard sphere repulsion}

In this paper we first consider a short-range potential $\Phi^{0}(R)$ in the form of hard sphere interaction,

$$
\Phi^{0}(R)=\left\{\begin{array}{c}
\infty, \quad R \leq a \\
0, \quad R>a
\end{array},\right.
$$

$a$ is the hard sphere diameter.

This leads to $S_{q}^{0}=S_{q}^{\mathrm{HS}}$, the structure factor of the hard sphere system. It was calculated from the pair distribution function of the hard sphere system in which we neglected all terms with orders of density higher than 2 . This approximation will be called here $\rho^{2}$-approximation or HS- $\rho^{2}$.

It is known that the Percus-Yevick approximation (PY) gives quite a good description in the case of shortrange interactions. It has also another advantage: there is an exact solution of the Ornstein-Zernike equation for the hard sphere system in PY [13]. We denote the structure factor obtained in this fashion as $S_{q}^{\mathrm{PY}}$.

The quantities $S_{q}^{\mathrm{HS}}-1$ and $S_{q}^{\mathrm{PY}}-1$ show a weak damping $\propto 1 / q^{2}$ at the large values of the wave vector. It leads to the complication of the calculation procedure since one should extend the integration limits in the Fourier transformation of $\nu_{q}$. More precisely speaking, the damping of $\nu_{q}$ was weak too and we used the extrapolation at large $q$ to obtain the correct behaviour of $\Phi(R)$ at small distances.

We have calculated the Fourier image $\nu_{q}$ and the potential $\Phi(R)$ using the hard sphere structure factor both in HS- $\rho^{2}$ and PY approximations. As one can see from Fig. 1, the long-range behaviour of this potential is not satisfactory unlike the short-range one.

One can see from Fig. 1 the extra hill on the potential curve at approximately $6 \AA$ A. We will try to find out due to what reasons it appears.
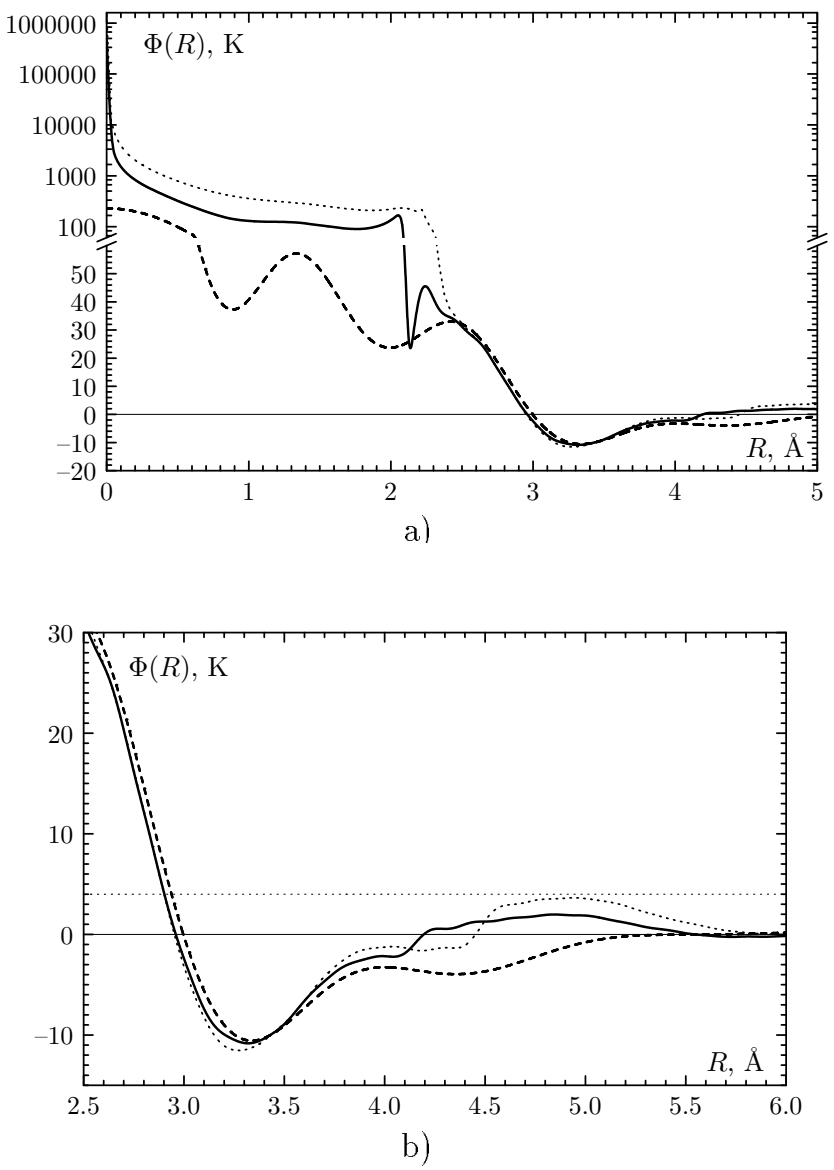

Fig. 1. The potential $\Phi(R)$ with short-range interactions in hard sphere approximation (HS- $\rho^{2}$ - solid line, PY dotted line) compared with results of our previous work [1] (dashed line).

\section{B. Softened repulsion}

The true repulsive part of the potential should be a bit "softened". It is easier to formulate this "softening" in terms of the Meyer function $f(R)$ :

$$
f(R)=e^{-\beta \Phi(R)}-1
$$

that stays finite while $\Phi(R)$ can have infinities, see (5).

For the hard sphere system $f(R)$ reads

$$
f^{\mathrm{HS}}(R)=\theta(R-a)-1=\left\{\begin{array}{cc}
-1, & R \leq a \\
0, & R>a
\end{array},\right.
$$

In these terms, the softening means that the straight step of the Meyer function of hard sphere must be slightly "rounded". Here we consider the variants of this "rounding".

Let us suppose the power dependence in $\Phi^{0}(R)$ : $\Phi^{0}(R) \propto 1 / R^{n}$. Then the kinetic energy operator in the Schrödinger equation gives: 


$$
\begin{aligned}
-\frac{\hbar^{2}}{2 m} \frac{\partial^{2}}{\partial R^{2}} e^{-(A / R)^{n}}= & -\frac{\hbar^{2}}{2 m} \frac{n^{2}}{R^{2}}\left(\frac{A}{R}\right)^{2 n} e^{-(A / R)^{n}} \\
& +O\left(1 / R^{n+2}\right)
\end{aligned}
$$

Thus, the power $2 n+2$ must equal the power of inverse distance in the repulsive part of real potential. Assuming it to be the Lennard-Jones potential we obtain $n=5$.

Another way is to round the corners in the Meyer function by directly accepting its form. For instance, like in the previous case:

$$
f(R)=e^{-(A / R)^{n}}-1
$$

The value of $n$ should be taken large (larger than 5) in order to resemble the hard sphere step. The parameter $A$ does not depend on the temparature unlike the exponent in expression (6) written for a classic system. The reason is that we consider a quantum system in the ground state and no temperature dependence is studied here.

The first case (Lennard-Jones repulsion, $n=5$ ) will be called "soft spheres" (SS), and the second one (directly accepted repulsion) will be denoted as "almost hard spheres" (AHS).

Let us choose the following values for AHS quantities in $(9)$ :

$$
A=2.1 \AA, \quad n=12 .
$$

The Meyer function then becomes quite close to the hard sphere step, see Fig. 2. That is the reason why we call it "almost hard spheres".

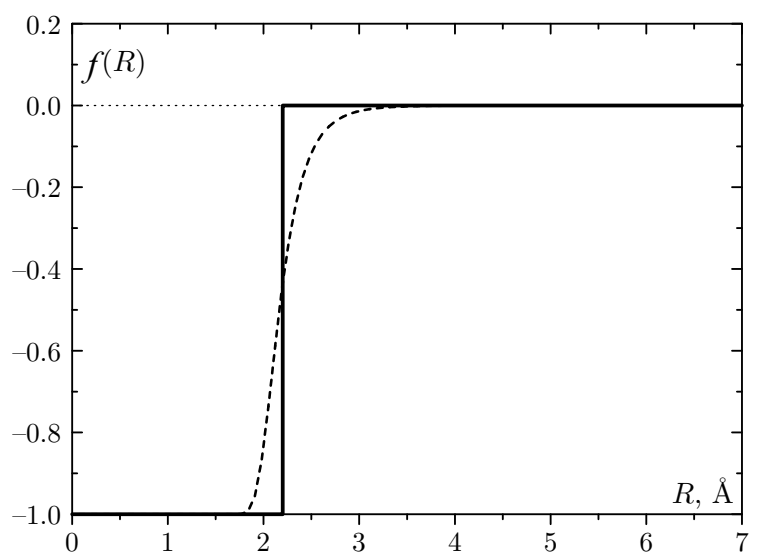

Fig. 2. Meyer functions of hard spheres (solid line) and almost hard spheres (dashed line).

We have calculated the structure factor of the softened repulsive part using the PY approximation. Since we do not need big accuracy, only the first iteration in the Ornstein-Zernike equation was used. Our results show that the potential curve does not differ much from that of our previous work [1], see Fig. 3 .

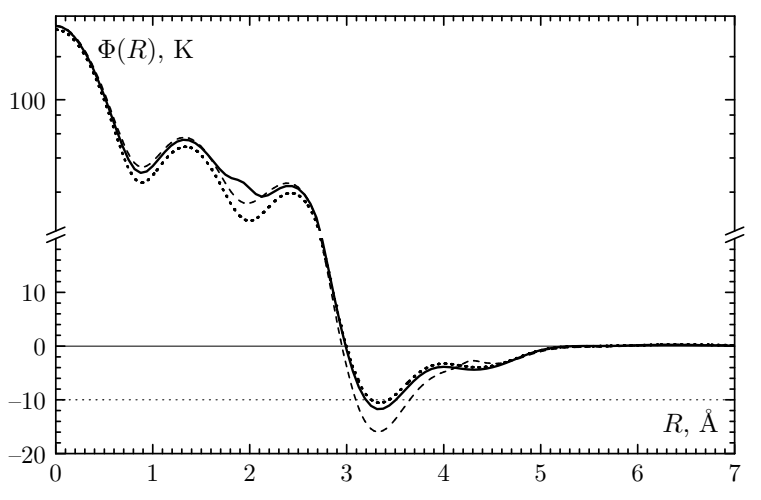

Fig. 3. The potential $\Phi(R)$ with short-range interactions in SS (dashed line) and AHS (solid line) approximation compared with results of our previous work [1] (dotted line).

In spite of a large difference in $1 / R$ powers ( 5 and 12 ), the results do not yield to a considerably different behaviour in the region of small $R$ : it still resembles that of [1]. From this point of view, the reason must lie in the fundamental difference between soft and hard spheres, namely in the analyticity or non-analyticity of the Meyer function that leads to different types of damping at large $q$ for the structure factor and, as a result, for the Fourier image $\nu_{q}$.

One other fact concerns the region of a potential well. One can see that the increasing of $1 / R$ power from 5 to 12 moves the depth of the well closer to that obtained from the hard sphere approximation. On the other hand, a similar value was obtained in [1] and it appears not to

\begin{tabular}{|c|c|c|c|}
\hline Potential & $\Phi(0), \mathrm{K}$ & Well & Extra Hill \\
\hline $\begin{array}{l}\text { Ref. } 1 \\
\text { HS }-\rho^{2} \\
\text { HS }- \text { PY } \\
\text { AHS } \\
\text { SS } \\
\text { Glued } \\
\end{array}$ & $\begin{aligned} \simeq & 240 \\
& \infty \\
& \infty \\
\simeq & 240 \\
\simeq & 230 \\
& \infty\end{aligned}$ & $\begin{array}{l}-10.59 \mathrm{~K} \text { at } 3.34 \AA \\
-10.86 \mathrm{~K} \text { at } 3.32 \AA \\
-11.55 \mathrm{~K} \text { at } 3.27 \AA \\
-11.76 \mathrm{~K} \text { at } 3.34 \AA \\
-15.95 \mathrm{~K} \text { at } 3.32 \AA \\
-11.72 \mathrm{~K} \text { at } 3.33 \AA\end{array}$ & $\begin{array}{l}0.26 \mathrm{~K} \text { at } 6.41 \AA \\
1.99 \mathrm{~K} \text { at } 4.84 \AA \\
3.64 \mathrm{~K} \text { at } 4.94 \AA \\
0.23 \mathrm{~K} \text { at } 6.31 \AA \\
0.27 \mathrm{~K} \text { at } 6.44 \AA \\
0.23 \mathrm{~K} \text { at } 6.31 \AA \\
\end{array}$ \\
\hline $\begin{array}{l}\text { Ref. } 6 \\
\text { Ref. } 5\end{array}$ & $2 \cdot 10^{6}$ & $\begin{array}{l}-11.01 \mathrm{~K} \text { at } 3.0 \AA \\
-11.04 \mathrm{~K} \text { at } 2.99 \AA\end{array}$ & $\begin{array}{l}\text { no hill } \\
\text { no hill }\end{array}$ \\
\hline
\end{tabular}
differ much from the results of computer simulations $[6,7]$ and the computations with adjustable parameters [2-5]. Those results might be found in Table 1.

Table 1. Parameters of the potential curve for different types of the potential.

The extra-hill was obtained on our potential curves at the interatomic distances of $\simeq 5-7 \AA$. We consider it as a consequence of solving the problem of interaction between helium atoms via quantum equations. But the large values of hill maxima obtained in the case of hard-sphere interaction should be treated as a result of the Meyer function non-analyticity. We claim this since the correspondent values in the case of soft spheres and almost hard spheres are commensurable with the value from [1]. 
The region of small $R$ values is probably the most complicated one. It seems that one should accept the hard-sphere approximation in order to obtain a good description here. We tried to join the advantages of the PY short-range part with those of the AHS long-range part by just "gluing" these two data sets at the point of $3.25 \AA$. The left side corresponds to PY; the right side to AHS.

Our values of the potential at small distances, namely for $R<2.5 \AA$, should not be considered as a correct quantitative result in this region but only as the qualitative one. What is done by accepting different models for short-range interaction are the data sets for the potential Fourier image $\nu_{q}$. In the case of hard spheres $\nu_{q}$ damps at large $q$ slowly giving a contribution into the potential as $\propto 1 / R$ after the Fourier transformation. If any softened repulsion is accepted the potential remains finite at $R=0 \AA$.

One can notice that there is a constant positive difference between the minimum position (the equilibrium interatomic distance) of the potentials discussed in this work and of those calculated by Aziz et al. [2-5]. We consider it as a consequence of the effective nature of our potentials. Let us consider the model of hard spheres with the diameter $a$ in order to illustrate this statement. The equilibrium distance between two atoms in the system of two atoms is obviously $a$. But if one considers the system of three particles then the average distance slightly increases, namely by 5 per cent. Thus, the difference of approximately 10 per cent in our case might be at least partially considered as a consequence of such a geometric effect.

\section{ENERGY AND SOUND VELOCITY}

One can calculate the potential energy $\Phi$ of the system at $0 \mathrm{~K}$ using relation [14]:

$$
\begin{aligned}
& \Phi=N \frac{\rho \nu_{0}}{2}+\sum_{\mathbf{q} \neq \mathbf{0}} \frac{\rho \nu_{q}}{2}\left(\frac{1}{\alpha_{q}}-1\right), \\
& \alpha_{q}=\sqrt{1+\frac{2 \rho \nu_{q}}{\varepsilon_{q}}}, \quad \varepsilon_{q}=\frac{\hbar^{2} q^{2}}{2 m} .
\end{aligned}
$$

The total energy $E$ reads:

$$
E=\Phi+\sum_{\mathbf{q} \neq \mathbf{0}} \frac{\varepsilon_{q}}{4} \frac{\left(\alpha_{q}-1\right)^{2}}{\alpha_{q}}
$$

The quantity $\rho \nu_{0}$ in these equation must be substituted with $m c^{2}$ where $c$ is the sound velocity in ${ }^{4} \mathrm{He}$. The value $m c^{2}=\rho \nu_{0}$ corresponds to the RPA and we want to have the next, postRPA approximation. Thus, the corrected expression reads [12]:

$$
m c^{2}=\rho \nu_{0}-\frac{1}{8 N} \sum_{\mathbf{q} \neq \mathbf{0}} \varepsilon_{q} \frac{\left(\alpha_{q}-1\right)^{2}}{\alpha_{q}} .
$$

We give the results discussed above in Table 1 for the sake of comparison. The left hand values of sound velocity correspond to the RPA $\left(m c^{2}=\rho \nu_{0}\right)$, while the right-hand values reflect the correction [12].

\begin{tabular}{|l|c|r|c|}
\hline Potential & $\Phi / N, \mathrm{~K}$ & $E / N, \mathrm{~K}$ & $c, \mathrm{~m} / \mathrm{s}$ \\
\hline Ref. 1 & -15.5 & -7.8 & 292 vs 238 \\
HS- $\rho^{2}$ a) & +18.1 & +38.8 & 407 vs 524 \\
AHS & -21.6 & -12.0 & 297 vs 240 \\
SS & -29.1 & -18.6 & 254 vs 182 \\
Glued & -16.7 & -8.0 & 324 vs 262 \\
\hline Ref. $8^{\mathrm{b}}$ ) & -21.7 & -7.1 & 241 \\
\hline Experiment & & $\left.-7.17^{\mathrm{c}}\right)$ & $\left.240.3^{\mathrm{d}}\right)$ \\
\hline
\end{tabular}

a) We do not give data for the potential obtained using $S^{\mathrm{PY}}$ since in this case one cannot obtain a "good" $\alpha_{q}$ : value of $1+2 \rho \nu_{q} / \varepsilon_{q}$ is negative for some values of $q$.

b) The calculation which uses the potential as in [2].

c) Ref. 10

d) This value corresponds to the sound velocity at saturated vapour pressure for $T=0.1 \mathrm{~K}$ [15]. Ref. 16 gives $237.8 \mathrm{~m} / \mathrm{s}$ at $T=0.8 \mathrm{~K}$. These values are not strictly experimental but it is possible to consider them as the averaged experimental ones since they are calculated using the well-analyzed equation of state.

Table 2. Energy and sound velocity with different potentials.

As might be seen from Table 2, the HS model for the short-range repulsion yields incorrect values for energy and sound velocity. Unfortunately, we did not find the universal potential satisfying the requirements to describe the total energy and the potential energy with similar accuracy. The best fit for both these quantities is found in the case of "glued" potential but the method of its obtaining looks very artificial. One can see that the best fit of the potential energy is found in the AHS approximation for the short-range repulsion. On the other hand, the previously obtained potential [1] remains the best of those considered here due to the values of the total energy and the sound velocity.

Therefore, three potentials will be used in our further works: the potential obtained in [1], the potential with AHS short-range repulsion and the "glued" potential. A comprehensive analysis of thermodynamic and transport properties that might be calculated and compared with the experiment or the computer simulations will determine the most suitable model potential. 
[1] I. O. Vakarchuk, V. V. Babin, A. A. Rovenchak, J. Phys. Stud. 4, 16 (2000).

[2] R. A. Aziz, V. P. S. Nain, J. S. Carley, W. L. Taylor, G. T. McConville, J. Chem. Phys. 70, 4430 (1979).

[3] R. A. Aziz, M. J. Slaman, J. Chem. Phys. 94, 8047 (1991).

[4] R. A. Aziz, M. J. Slaman, A. Koide, A. R. Allnatt, W. J. Meath, Mol. Phys. 77, 321 (1992).

[5] A. R. Janzen and R. A. Aziz, J. Chem. Phys. 107, 914 (1997).

[6] J. A. Anderson, C. A. Traynor, B. M. Boghosian, J. Chem. Phys. 99, 345 (1993).

[7] K. T. Tang, J. P. Toennies, C. L. Yiu, Phys. Rev. B 74, 1546 (1995).

[8] J. Boronat, J. Casulleras, Phys. Rev. B 49, 8920 (1994).
[9] Svensson E. C., Sears V. F., Woods A. D. B., Martel P. Phys. Rev. B, 21, 8 (1980).

[10] R. De Bruyn Ouboter, C.N. Yang, Physica B 44, 127 (1987).

[11] I. A. Vakarchuk, Teor. Mat. Fiz. 80, 439 (1989); 82, 438 (1990).

[12] I. A. Vakarchuk, I. R. Yukhnovsky, Teor. Mat. Fiz. 40, 100 (1979).

[13] R. Balescu, Equilibrium and nonequilibrium statistical mechanics (New York: Wiley, 1975).

[14] I. O. Vakarchuk, J. Phys. Stud. 1, 25 (1996).

[15] R. D. McCarthy, Nat. Bur. Stand. (U.S.), Tech. Note 1029, (1980).

[16] V. D. Arp, R. D. McCarty, D. G. Friend, Natl. Inst. Stand. Technol. Tech. Note 1334 (revised) (1998).

\section{ПРЕДСТАВЛЕННЯ ВЗАЄМОДІЇ НА МАЛИХ ВІДДАЛЯХ У РІДКОМУ ГЕЛІЇ ЗА ДОПОМОГОЮ МОДИФІКОВАНИХ ПОТЕНЩЯЛІВ ТВЕРДИХ СФЕР}

I. О. Вакарчук, А. А. Ровенчак

Львівський начіональний університет імені Івана Франка, кафедра теоретичної фізики вул. Драгоманова, 12, Львів, 79005, Україна

\footnotetext{
Запропоновано п'ять різних модифікапій потенпіялу твердих сфер для моделювання відштовхування на коротких віддалях й обчислення термодинамічних $\mathrm{i}$ структурних функпій рі,дкого ${ }^{4}$ Не. Розраховано потенпіяльну енергію, повну енергію та швидкість звуку при $T=0 \mathrm{~K}$. Показано, що три із запропонованих потенціялів дають задовільний опис цих величин.
} 